\title{
Strangeness and phase changes in hot hadronic matter - 1983
}

\author{
From: "Sixth High Energy Heavy Ion Study" \\ held 28 June - 1 July 1983 at: LBNL, Berkeley, CA, USA \\ Johann Rafelski ${ }^{1,2, a, b}$ \\ 1 CERN-PH/TH, 1211 Geneva 23, Switzerland \\ 2 Department of Physics, The University of Arizona Tucson, Arizona, 85721, USA
}

Addendum to: Eur. Phys. J. A (2015) 51: 114, DOI: 10.1140/epja/i2015-15114-0

Received: 12 August 2015 / Revised: 23 August 2015

Published online: 22 September 2015

(c) The Author(s) 2015. This article is published with open access at Springerlink.com

Communicated by T.S. Bíró

\begin{abstract}
Two phases of hot hadronic matter are described with emphasis put on their distinction. Here the role of strange particles as a characteristic observable of the quark-gluon plasma phase is particularly explored.
\end{abstract}

\section{Phase transition or perhaps transformation: Hadronic gas and the quark-gluon plasma}

I explore here consequences of the hypothesis that the energy available in the collision of two relativistic heavy nuclei, at least in part of the system, is equally divided among the accessible degrees of freedom. This means that there exists a domain in space in which, in a suitable Lorentz frame, the energy of the longitudinal motion has been largely transformed to transverse degrees of freedom. The physical variables characterizing such a "fireball" are energy density, baryon number density, and total volume. The basic question concerns the internal structure of the fireball. It can consist either of individual hadrons, or instead, of quarks and gluons in a new physical phase, the plasma, in which they are deconfined and can move freely over the volume of the fireball. It appears that the phase transition from the hadronic gas phase to the quark-gluon plasma is controlled mainly by the energy density of the fireball. Several estimates ${ }^{1}$ lead to $0.6-1 \mathrm{GeV} / \mathrm{fm}^{3}$ for the critical energy density, to be compared with nuclear matter $0.16 \mathrm{GeV} / \mathrm{fm}^{3}$.

${ }^{a}$ The original address byline 1983: CERN, Genva, Switzerland and Institut für Theoretische Physics der Universität Frankfurt/M; originally printed in LBL-16281 pp. 489-510; also: report number UC-34C; DOE CONF-830675; preprint CERN-TH-3685 available at https://cds.cern.ch/record/ 147343/files/198311019.pdf.

b e-mail: rafelski@physics.arizona.edu

${ }^{1}$ An incomplete list of quark-gluon plasma papers includes [1-10].
We first recall that the unhandy extensive variables, viz., energy, baryon number, etc., are replaced by intensive quantities. To wit, the temperature $T$ is a measure of energy per degree of freedom; the baryon chemical potential $\mu$ controls the mean baryon density. The statistical quantities such as entropy (= measure of the number of available states), pressure, heat capacity, etc., will also be functions of $T$ and $\mu$, and will have to be determined. The theoretical techniques required for the description of the two quite different phases, viz., the hadronic gas and the quark-gluon plasma, must allow for the formulation of numerous hadronic resonances on the one side, which then at sufficiently high energy density dissolve into the state consisting of their constituents ${ }^{2}$. At this point, we must appreciate the importance and help by a finite, i.e., nonzero temperature in reaching the transition to the quark-gluon plasma: to obtain a high particle density, instead of only compressing the matter (which as it turns out is quite difficult), we also heat it up; many pions are generated in a collision, allowing the transition to occur at moderate, even vanishing baryon density [14].

Consider, as an illustration of what is happening, the $p, V$ diagram shown in fig. 1 . Here we distinguish three domains. The hadronic gas region is approximately a Boltzmann gas where the pressure rises with reduction of the volume. When the internal excitation rises, the individual hadrons begin to cluster. This reduces the increase in the Boltzmann pressure, since a smaller number of particles exercises a smaller pressure. In a complete description of the different phases, we have to allow for a coexistence of

\footnotetext{
2 These ideas originate in Hagedorn's statistical bootstrap theory [11-13].
} 


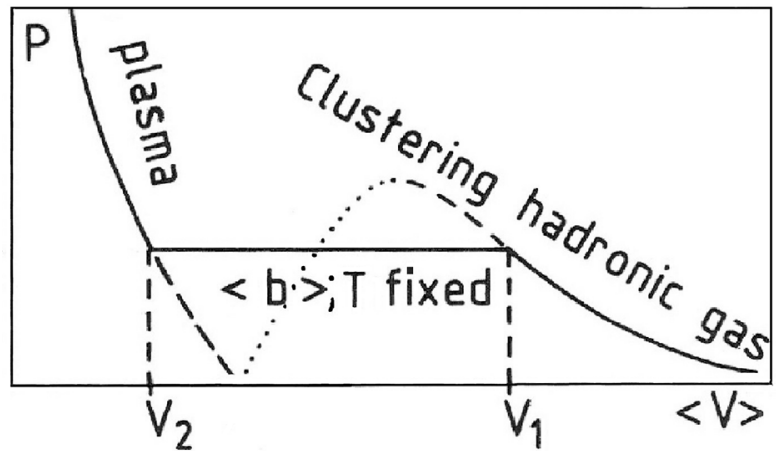

Fig. 1. $p-V$ diagram for the gas-plasma first order transition, with the dotted curve indicating a model-dependent, unstable domain between overheated and undercooled phases.

hadrons with the plasma state in the sense that the internal degrees of freedom of each cluster, i.e., quarks and gluons, contribute to the total pressure even before the dissolution of individual hadrons. This does indeed become necessary when the clustering overtakes the compressive effects and the hadronic gas pressure falls to zero as $V$ reaches the proper volume of hadronic matter. At this point the pressure rises again very quickly, since in the absence of individual hadrons, we now compress only the hadronic constituents. By performing the Maxwell construction between volumes $V_{1}$ and $V_{2}$, we can in part account for the complex process of hadronic compressibility alluded to above.

As this discussion shows, and detailed investigations confirm [15-18], we cannot escape the conjecture of a first order phase transition in our approach. This conjecture of [8] has been criticized, and only more recent lattice gauge theory calculations have led to the widespread acceptance of this phenomenon, provided that an internal $\mathrm{SU}(3)$ (color) symmetry is used - SU(2) internal symmetry leads to a second order phase transition [10]. It is difficult to assess how such hypothetical changes in actual internal particle symmetry would influence phenomenological descriptions based on an observed picture of nature. For example, it is difficult to argue that, were the color symmetry SU(2) and not SU(3), we would still observe the resonance dominance of hadronic spectra and could therefore use the bootstrap model. All present understanding of phases of hadronic matter is based on approximate models, which requires that table 1 be read from left to right.

I believe that the description of hadrons in terms of bound quark states on the one hand, and the statistical bootstrap for hadrons on the other hand, have many common properties and are quite complementary. Both the statistical bootstrap and the bag model of quarks are based on quite equivalent phenomenological observations. While it would be most interesting to derive the phenomenological models quantitatively from the accepted fundamental basis - the Lagrangian quantum field theory of a non-Abelian SU(3) "glue" gauge field coupled to colored quarks - we will have to content ourselves in this report with a qualitative understanding only. Already this will allow us to study the properties of hadronic matter in both aggregate states: the hadronic gas and the state in which individual hadrons have dissolved into the plasma consisting of quarks and of the gauge field quanta, the gluons.

It is interesting to follow the path taken by an isolated quark-gluon plasma fireball in the $\mu, T$ plane, or equivalently in the $\nu, T$ plane. Several cases are depicted in fig. 2. In the Big Bang expansion, the cooling shown by the dashed line occurs in a Universe in which most of the energy is in the radiation. Hence, the baryon density $\nu$ is quite small. In normal stellar collapse leading to cold neutron stars, we follow the dash-dotted line parallel to the $\nu$ axis. The compression is accompanied by little heating.

In contrast, in nuclear collisions, almost the entire $\nu, T$ plane can be explored by varying the parameters of the colliding nuclei. We show an example by the full line, and we show only the path corresponding to the cooling of the plasma, i.e., the part of the time evolution after the termination of the nuclear collision, assuming a plasma formation. The figure reflects the circumstance that, in the beginning of the cooling phase, i.e., for $1-1.5 \times 10^{-23} \mathrm{~s}$, the cooling happens almost exclusively by the mechanism of pion radiation $[19,20]$. In typical circumstances, about half of the available energy has been radiated away before the expansion, which brings the surface temperature close to the temperature of the transition to the hadronic phase. Hence a possible, perhaps even likely, scenario is that in which the freezing out and the expansion happen simultaneously. These highly speculative remarks are obviously made in the absence of experimental guidance. A careful study of the hadronization process most certainly remains to be performed.

In closing this section, let me emphasize that the question whether the transition hadronic gas $\longleftrightarrow$ quark-gluon plasma is a phase transition (i.e., discontinuous) or continuous phase transformation will probably only be answered in actual experimental work; as all theoretical approaches suffer from approximations unknown in their effect. For example, in lattice gauge computer calculations, we establish the properties of the lattice and not those of the continuous space in which we live.

The remainder of this report is therefore devoted to the study of strange particles in different nuclear phases and their relevance to the observation of the quark-gluon plasma.

\section{Strange particles in hot nuclear gas}

My intention in this section is to establish quantitatively the different channels in which the strangeness, however created in nuclear collisions, will be found. In our following analysis (see ref. [21]) a tacit assumption is made that the hadronic gas phase is practically a superposition of an infinity of different hadronic gases, and all information about the interaction is hidden in the mass spectrum $\tau\left(m^{2}, b\right)$ which describes the number of hadrons of baryon number $b$ in a mass interval $\mathrm{d}^{2}$ and volume $V \sim m$. When considering strangeness-carrying particles, all we then need to include is the influence of the non-strange 
Table 1. Phase transition of hot hadronic matter in theoretical physics.

\begin{tabular}{|lllll|}
\hline Object & $\longrightarrow$ & Observational hypothesis & $\longrightarrow$ & Theoretical consequence \\
\hline Nature & $\longrightarrow$ & Internal SU(3) symmetry & $\longrightarrow$ & $\begin{array}{l}\text { First order phase transition } \\
\text { (on a lattice) }\end{array}$ \\
\hline Nature & $\longrightarrow$ & $\begin{array}{l}\text { Bootstrap } \text { = resonance } \\
\text { dominance of hadronic } \\
\text { interactions }\end{array}$ & $\longrightarrow$ & $\begin{array}{l}\text { First order phase transition } \\
\text { in a phenomenological } \\
\text { bootstrap approach }\end{array}$ \\
\hline$?$ & $\longrightarrow$ & Internal SU(2) symmetry & $\longrightarrow$ & $\begin{array}{l}\text { Second order phase transition } \\
\text { (on a lattice) }\end{array}$ \\
\hline
\end{tabular}

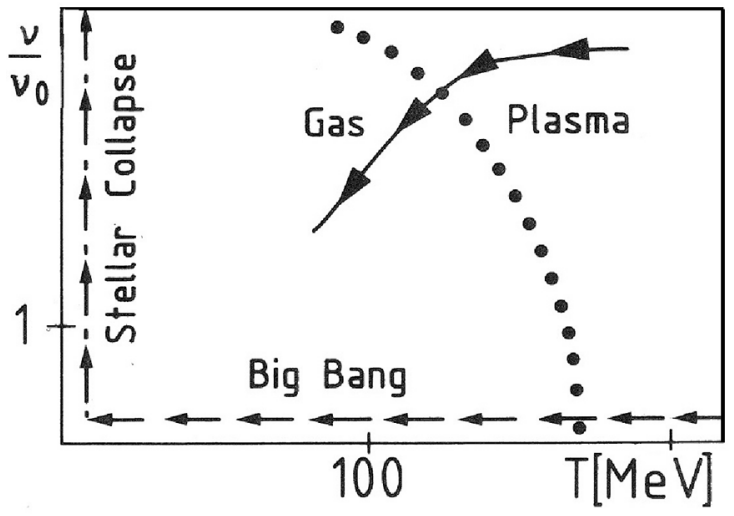

Fig. 2. Paths taken in the $\nu, T$ plane by different physical events.

hadrons on the baryon chemical potential established by the non-strange particles.

The total partition function is approximately multiplicative in these degrees of freedom:

$$
\ln Z=\ln Z^{\text {non-strange }}+\ln Z^{\text {strange }} .
$$

For our purposes, i.e., in order to determine the particle abundances, it is sufficient to list the strange particles separately, and we find

$$
\begin{aligned}
& \ln Z^{\text {strange }}\left(T, V, \lambda_{s}, \lambda_{q}\right)= \\
& C\left\{2 W\left(x_{\mathrm{K}}\right)\left(\lambda_{s} \lambda_{q}^{-1}+\lambda_{s}^{-1} \lambda_{q}\right)\right. \\
& \left.+2\left[W\left(x_{\Lambda}\right)+3 W\left(x_{\Sigma}\right)\right]\left(\lambda_{s} \lambda_{q}^{2}+\lambda_{s}^{-1} \lambda_{q}^{-2}\right)\right\},
\end{aligned}
$$

where

$$
W\left(x_{i}\right)=\left(\frac{m_{i}}{T}\right)^{2} K_{2}\left(\frac{m_{i}}{T}\right) .
$$

We have $C=V T^{3} / 2 \pi^{2}$ for a fully equilibrated state. However, strangeness-creating $(x \rightarrow s+\bar{s})$ processes in hot hadronic gas may be too slow (see below) and the total abundance of strange particles may fall short of this value of $C$ expected in absolute strangeness chemical equilibrium. On the other hand, strangeness exchange cross-sections are very large (e.g., the $K^{-} p$ cross-section is $\sim 100 \mathrm{mb}$ in the momentum range of interest), and therefore any momentarily available strangeness will always be distributed among all particles in eq. (2.2) according to the values of the fugacities $\lambda_{q}=\lambda_{\mathrm{B}}^{1 / 3}$ and $\lambda_{s}$. Hence we can speak of a relative strangeness chemical equilibrium.

We neglected to write down quantum statistics corrections as well as the multistrange particles $\Xi$ and $\Omega^{-}$, as our considerations remain valid in this simple approximation [22]. Interactions are effectively included through explicit reference to the baryon number content of the strange particles, as just discussed. Non-strange hadrons influence the strange faction by establishing the value of $\lambda_{q}$ at the given temperature and baryon density.

The fugacities $\lambda_{s}$ and $\lambda_{q}$ as introduced here control the strangeness and the baryon number, respectively. While $\lambda_{s}$ counts the strange quark content, the up and down quark content is counted by $\lambda_{q}=\lambda_{\mathrm{B}}^{1 / 3}$.

Using the partition function eq. (2.2), we calculate for given $\mu, T$, and $V$ the mean strangeness by evaluating

$$
\left\langle n_{s}-n_{\bar{s}}\right\rangle=\lambda_{s} \frac{\partial}{\partial \lambda_{s}} \ln Z^{\text {strange }}\left(T, V, \lambda_{s}, \lambda_{q}\right)
$$

which is the difference between strange and antistrange components. This expression must be equal to zero due to the fact that the strangeness is a conserved quantum number with respect to strong interactions. From this condition, we get $^{3}$

$$
\lambda_{s}=\lambda_{q}\left|\frac{W\left(x_{\mathrm{K}}\right)+\lambda_{\mathrm{B}}^{-1}\left[W\left(x_{\Lambda}\right)+3 W\left(x_{\Sigma}\right)\right]}{W\left(x_{\mathrm{K}}\right)+\lambda_{\mathrm{B}}\left[W\left(x_{\Lambda}\right)+3 W\left(x_{\Sigma}\right)\right]}\right|^{1 / 2} \equiv \lambda_{q} F,
$$

a result contrary to intuition: $\lambda_{s} \neq 1$ for a gas with total $\langle s\rangle=0$. We notice a strong dependence of $F$ on the baryon number. For large $\mu$, the term with $\lambda_{\mathrm{B}}^{-1}$ will tend to zero and the term with $\lambda_{\mathrm{B}}$ will dominate the expression for $\lambda_{s}$ and $F$. As a consequence, the particles with fugacity $\lambda_{s}$ and strangeness $S=-1$ (note that by convention strange quarks $s$ carry $S=-1$, while strange antiquarks $\bar{s}$ carry $S=1$ ) are suppressed by a factor $F$ which is always smaller than unity. Conversely, the production of particles which carry the strangeness $S=+1$ will be favored by $F^{-1}$. This is a consequence of the presence of nuclear matter: for $\mu=0$, we find $F=1$.

In nuclear collisions, the mutual chemical equilibrium, that is, a proper distribution of strangeness among the strange hadrons, will most likely be achieved. By studying the relative yields, we can exploit this fact and eliminate the absolute normalization $C$ (see eq. (2.2)) from our considerations. We recall that the value of $C$ is uncertain for several reasons:

i) $V$ is unknown.

${ }^{3}$ Notation has been changed $\gamma \rightarrow F$ in order to avoid confusion with phase space occupancy $\gamma$. 
ii) $C$ is strongly $(t, r)$-dependent, through the space-time dependence of $T$.

iii) Most importantly, the value $C=V T^{3} / 2 \pi^{2}$ assumes absolute chemical equilibrium, which is not achieved owing to the shortness of the collision.

Indeed, we have (see eq. (4.3) for in plasma strangeness formation and further details and solutions)

$$
\frac{\mathrm{d} C}{\mathrm{~d} t}=A_{\mathrm{H}}\left[1-\frac{C(t)^{2}}{C(\infty)^{2}}\right],
$$

and the time constant for strangeness production in nuclear matter can be estimated to be [23]

$$
\tau_{\mathrm{H}}=C(\infty) / 2 A_{\mathrm{H}} \sim 10^{-21} \mathrm{~s}
$$

Thus $C$ does not reach $C(\infty)$ in plasmaless nuclear collisions. If the plasma state is formed, then the relevant $C>C(\infty)$ (since strangeness yield in plasma is above strangeness yield in hadron gas (see below).

Now, why should we expect relative strangeness equilibrium to be reached faster than absolute strangeness equilibrium [21]? Consider the strangeness exchange interaction

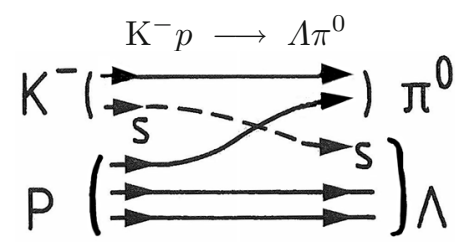

which has a cross-section of about $10 \mathrm{mb}$ at low energies, while the $s \bar{s}$ "strangeness creating" associate production

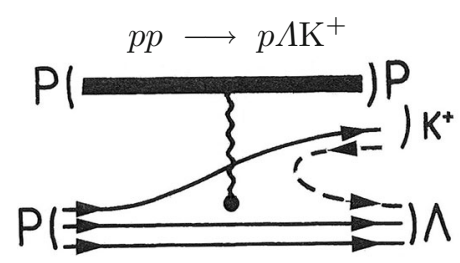

has a cross-section of less than $0.06 \mathrm{mb}$, i.e., 150 times smaller. Since the latter reaction is somewhat disfavored by phase space, consider further the reaction

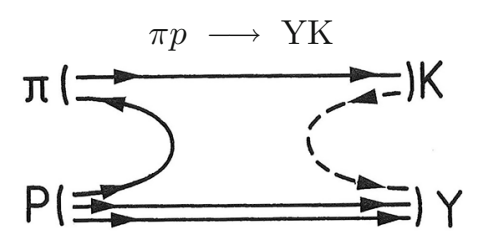

where $\mathrm{Y}$ is any hyperon (strange baryon). This has a crosssection of less than $1 \mathrm{mb}$, still 10 times weaker than one of the s-exchange channels in eq. (2.7). Consequently, I expect the relative strangeness equilibration time to be about ten times shorter than the absolute strangeness equilibration time, namely $10^{-23} \mathrm{~s}$, in hadronic matter of about twice nuclear density.

We now compute the relative strangeness abundances expected from nuclear collisions. Using eq. (2.5), we find

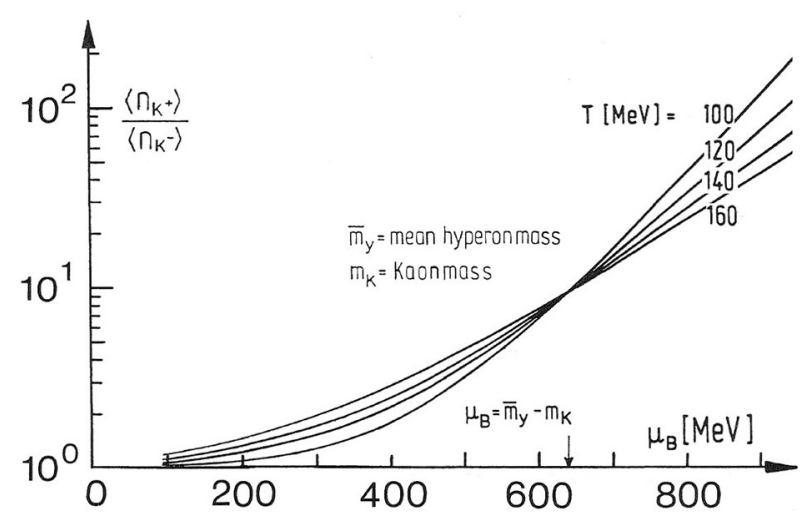

Fig. 3. The ratio $\left\langle n_{\mathrm{K}^{+}}\right\rangle /\left\langle n_{\mathrm{K}^{-}}\right\rangle \equiv F^{-2}$ as a function of the baryon chemical potential $\mu$, for $T=100,(20), 160 \mathrm{MeV}$. The lines cross where $\mu=\bar{m}_{\mathrm{Y}}-m_{\mathrm{K}} ; \bar{m}_{\mathrm{Y}}$ is the mean hyperon mass.

from eq. (2.2) the grand canonical partition sum for zero average strangeness

$$
\begin{aligned}
& \ln Z_{0}^{\text {strange }} C\left[2 W\left(x_{\mathrm{K}}\right)\left(F \lambda_{\mathrm{K}}+F^{-1} \lambda_{\overline{\mathrm{K}}}\right)\right. \\
& +2 W\left(x_{\Lambda}\right)\left(F \lambda_{\mathrm{B}} \lambda_{\Lambda}+F^{-1} \lambda_{\mathrm{B}}^{-1} \lambda_{\bar{\Lambda}}\right) \\
& \left.+6 W\left(x_{\Sigma}\right)\left(F \lambda_{\mathrm{B}} \lambda_{\Sigma}+F^{-1} \lambda_{\mathrm{B}}^{-1} \lambda_{\bar{\Sigma}}\right)\right],
\end{aligned}
$$

where, in order to distinguish different hadrons, dummy fugacities $\lambda_{i}, i=\mathrm{K}, \overline{\mathrm{K}}, \Lambda, \bar{\Lambda}, \Sigma, \bar{\Sigma}$ have been written. The strange particle multiplicities then follow from

$$
\left\langle n_{i}\right\rangle=\left.\lambda_{i} \frac{\partial}{\partial \lambda_{i}} \ln Z_{0}^{\text {strange }}\right|_{\lambda_{i}=1} .
$$

Explicitly, we find (notice that the power of $F$ follows the $s$-quark content):

$$
\begin{aligned}
\left\langle n_{\mathrm{K}^{ \pm}}\right\rangle & =C F^{\mp} W\left(x_{\mathrm{K}}\right), \\
\left\langle n_{\Lambda / \Sigma^{0}}\right\rangle & =C F^{+1} W\left(x_{\Lambda / \Sigma^{0}}\right) \mathrm{e}^{+\mu_{\mathrm{B}} / T}, \\
\left\langle n_{\bar{\Lambda} / \bar{\Sigma}^{0}}\right\rangle & =C F^{-1} W\left(x_{\bar{\Lambda} / \bar{\Sigma}^{0}}\right) \mathrm{e}^{-\mu_{\mathrm{B}} / T} .
\end{aligned}
$$

In eq. (2.14) we have indicated that the multiplicity of antihyperons can only be built up if antibaryons are present according to their (small) phase space. This still seems an unlikely proposition, and the statistical approach may be viewed as providing an upper limit on their multiplicity.

From the above equations, we can derive several very instructive conclusions. In fig. 3 we show the ratio

$$
\left\langle n_{\mathrm{K}^{+}}\right\rangle /\left\langle n_{\mathrm{K}^{-}}\right\rangle=F^{-2}
$$

as a function of the baryon chemical potential $\mu$ for several temperatures that can be expected and which are seen experimentally. We see that this particular ratio is a good measure of the baryon chemical potential in the hadronic gas phase, provided that the temperatures are approximately known. The mechanism for this process is as follows: the strangeness exchange reaction of eq. (2.7) tilts to the left $\left(\mathrm{K}^{-}\right)$or to the right (abundance $F \sim \mathrm{K}^{+}$), depending on the value of the baryon chemical potential.

In fig. 4 the long dashed line shows the upper limit for the abundance of $\bar{\Lambda}$ as measured in terms of $\Lambda$ abundances. 


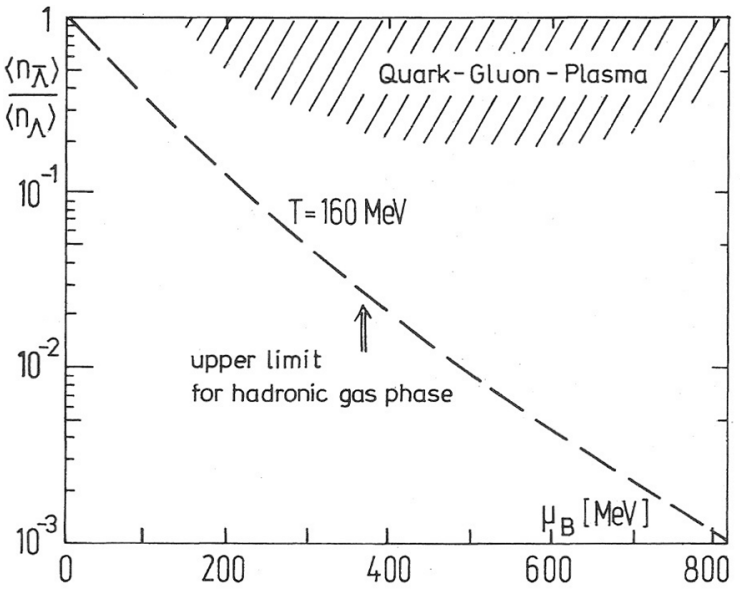

Fig. 4. Relative abundance of $\bar{\Lambda} / \Lambda$. The actual yield from the hadronic gas limit may still be 10-100 times smaller than the statistical value shown.

Clearly visible is the substantial relative suppression of $\bar{\Lambda}$, in part caused by the baryon chemical potential factor of eq. (2.14), but also by the strangeness chemistry (factor $F^{2}$ ), as in $\mathrm{K}^{+} \mathrm{K}^{-}$above. Indeed, the actual relative number of $\bar{\Lambda}$ will be even smaller, since $\Lambda$ are in relative chemical equilibrium and $\bar{\Lambda}$ in hadron gas are not: the reaction $\mathrm{K}^{+} \bar{p} \rightarrow \bar{\Lambda} \pi^{0}$, analogue to eq. (2.7), will be suppressed by low $\bar{p}$ abundance. Also indicated in fig. 4 by shading is a rough estimate for the $\bar{\Lambda}$ production in the plasma phase, which suggests that anomalous $\bar{\Lambda}$ abundance may be an interesting feature of highly energetic nuclear collisions [35], for further discussion see sect. 5 below.

\section{Quark-gluon plasma}

From the study of hadronic spectra, as well as from hadron-hadron and hadron-lepton interactions, there has emerged convincing evidence for the description of hadronic structure in terms of quarks [24]. For many purposes it is entirely satisfactory to consider baryons as bound states of three fractionally charged particles, while mesons are quark-antiquark bound states. The Lagrangian of quarks and gluons is very similar to that of electrons and photons, except for the required summations over flavour and color:

$$
L=\bar{\psi}[F \cdot(p-g A)-m] \psi-\frac{1}{4} F_{\mu \nu} F^{\mu \nu} .
$$

The flavour-dependent masses $m$ of the quarks are small. For $u, d$ flavours, one estimates $m_{u, d} \sim 5-20 \mathrm{MeV}$. The strange quark mass is usually chosen at about $150 \mathrm{MeV}$ $[25,26]$. The essential new feature of QCD, not easily visible in eq. (3.1), is the non-linearity of the field strength $F$ in terms of the potentials $A$. This leads to an attractive glue-glue interaction in select channels and, as is believed, requires an improved (non-perturbative) vacuum state in which this interaction is partially diagonalized, providing for a possible perturbative approach.

The energy density of the perturbative vacuum state, defined with respect to the true vacuum state, is by definition a positive quantity, denoted by $\mathcal{B}$. This notion has been introduced originally in the MIT bag model [27-29], logically, e.g., from a fit to the hadronic spectrum, which gives

$$
\mathcal{B}=[(140-210) \mathrm{MeV}]^{4}=(50-250) \mathrm{MeV} / \mathrm{fm}^{3} .
$$

The central assumption of the quark bag approach is that, inside a hadron where quarks are found, the true vacuum structure is displaced or destroyed. One can turn this point around: quarks can only propagate in domains of space in which the true vacuum is absent. This statement is a reformulation of the quark confinement problem. Now the remaining difficult problem is to show the incompatibility of quarks with the true vacuum structure. Examples of such behavior in ordinary physics are easily found; e.g., a light wave is reflected from a mirror surface, magnetic field lines are expelled from superconductors, etc. In this picture of hadronic structure and quark confinement, all colorless assemblies of quarks, antiquarks, and gluons can form stationary states, called a quark bag. In particular, all higher combinations of the three-quark baryons $(q q q)$ and quark-antiquark mesons $(q \bar{q})$ form a permitted state.

As the $u$ and $d$ quarks are almost massless inside a bag, they can be produced in pairs, and at moderate internal excitations, i.e., temperatures, many $q \bar{q}$ pairs will be present. Similarly, $s \bar{s}$ pairs will also be produced. We will return to this point at length below. Furthermore, real gluons can be excited and will be included here in our considerations.

Thus, what we are considering here is a large quark bag with substantial, equilibrated internal excitation, in which the interactions can be handled (hopefully) perturbatively. In the large volume limit, which as can be shown is valid for baryon number $b \gtrsim 10$, we simply have for the light quarks the partition function of a Fermi gas which, for practically massless $u$ and $d$ quarks can be given analytically (see ref. [2] and [30,31]), even including the effects of interactions through first order in $\alpha_{s}=g^{2} / 4 \pi$ :

$$
\begin{aligned}
\ln Z_{q}(\beta, \mu)= & \frac{g V}{6 \pi^{2}} \beta^{-3}\left\{\left(1-\frac{2 \alpha_{s}}{\pi}\right)\left[\frac{1}{4}(\mu \beta)^{4}+\frac{\pi^{2}}{2}(\mu \beta)^{2}\right]\right. \\
& \left.+\left(1-\frac{50}{21} \frac{\alpha_{s}}{\pi}\right) \frac{7 \pi^{4}}{60}\right\}
\end{aligned}
$$

Similarly, the glue is a Bose gas

$$
\ln Z_{g}(\beta, \lambda)=V \frac{8 \pi^{2}}{45} \beta^{-3}\left(1-\frac{15}{4} \frac{\alpha_{s}}{\pi}\right),
$$

while the term associated with the difference to the true vacuum, the bag term, is

$$
\ln Z_{\mathrm{bag}}=-\mathcal{B} V \beta \text {. }
$$

It leads to the required positive energy density $\mathcal{B}$ within the volume occupied by the colored quarks and gluons and to a negative pressure on the surface of this region. At this stage, this term is entirely phenomenological, as discussed above. The equations of state for the quark-gluon plasma are easily obtained by differentiating

$$
\ln Z=\ln Z_{q}+\ln Z_{g}+\ln Z_{\mathrm{vac}},
$$

with respect to $\beta, \mu$, and $V$. 
An assembly of quarks in a bag will assume a geometric shape and size such as to make the total energy $E(V, b, S)$ as small as possible at fixed given baryon number and fixed total entropy $S$. Instead of just considering one bag we may, in order to be able to use the methods of statistical physics, use the microcanonical ensemble. We find from the first law of thermodynamics, viz.

$$
\mathrm{d} E=-P \mathrm{~d} V+T \mathrm{~d} S+\mu \mathrm{d} b,
$$

that

$$
P=-\frac{\partial E(V, b, S)}{\partial V} .
$$

We observe that the stable configuration of a single bag, viz., $\partial E / \partial V=0$, corresponds to the configuration with vanishing pressure $P$ in the microcanonical ensemble. Rather than work in the microcanonical ensemble with fixed $b$ and $S$, we exploit the advantages of the grand canonical ensemble and consider $P$ as a function of $\mu$ and $T$ :

$$
P=-\frac{\partial}{\partial V}[T \ln Z(\mu, T, V)]
$$

with the result

$$
P=\frac{1}{3}(\varepsilon-4 \mathcal{B})
$$

where $\varepsilon$ is the energy density:

$$
\begin{aligned}
\varepsilon= & \frac{6}{\pi^{2}}\left\{\left(1-\frac{2 \alpha_{s}}{\pi}\right)\left[\frac{1}{4}\left(\frac{\mu}{3}\right)^{4}+\frac{1}{2}\left(\frac{\mu}{3}\right)^{2}(\pi T)^{2}\right]\right. \\
& \left.+\left(1-\frac{50}{21} \frac{\alpha_{s}}{\pi}\right) \frac{7}{60}(\pi T)^{4}\right\} \\
& +\left(1-\frac{15}{4} \frac{\alpha_{s}}{\pi}\right) \frac{8}{15 \pi^{2}}(\pi T)^{4}+\mathcal{B} .
\end{aligned}
$$

In eq. (3.10), we have used the relativistic relation between the quark and gluon energy density and pressure:

$$
P_{q}=\frac{1}{3} \varepsilon_{q}, \quad P_{g}=\frac{1}{3} \varepsilon_{g} .
$$

From eq. (3.10), it follows that, when the pressure vanishes in a static configuration, the energy density is $4 \mathcal{B}$, independently of the values of $\mu$ and $T$ which fix the line $P=0$. We note that, in both quarks and gluons, the interaction conspires to reduce the effective available number of degrees of freedom. At $\alpha_{s}=0, \mu=0$, we find the handy relation

$$
\varepsilon_{q}+\varepsilon_{g}=\left(\frac{T}{160 \mathrm{MeV}}\right)^{4}\left[\frac{\mathrm{GeV}}{\mathrm{fm}^{3}}\right] .
$$

It is important to appreciate how much entropy must be created to reach the plasma state. From eq. (3.6), we find for the entropy density $\mathcal{S}$ and the baryon density $\nu$

$$
\begin{aligned}
\mathcal{S}= & \frac{2}{\pi}\left(1-\frac{2 \alpha_{s}}{\pi}\right)\left(\frac{\mu}{3}\right)^{2} \pi T+\frac{14}{15 \pi}\left(1-\frac{50}{21} \frac{\alpha_{s}}{\pi}\right)(\pi T)^{3} \\
& +\frac{32}{45 \pi}\left(1-\frac{15}{4} \frac{\alpha_{s}}{\pi}\right)(\pi T)^{3} \\
\nu= & \frac{2}{3 \pi^{2}}\left\{\left(1-\frac{2 \alpha_{s}}{\pi}\right)\left[\left(\frac{\mu}{3}\right)^{3}+\frac{\mu}{3}(\pi T)^{2}\right]\right\}
\end{aligned}
$$
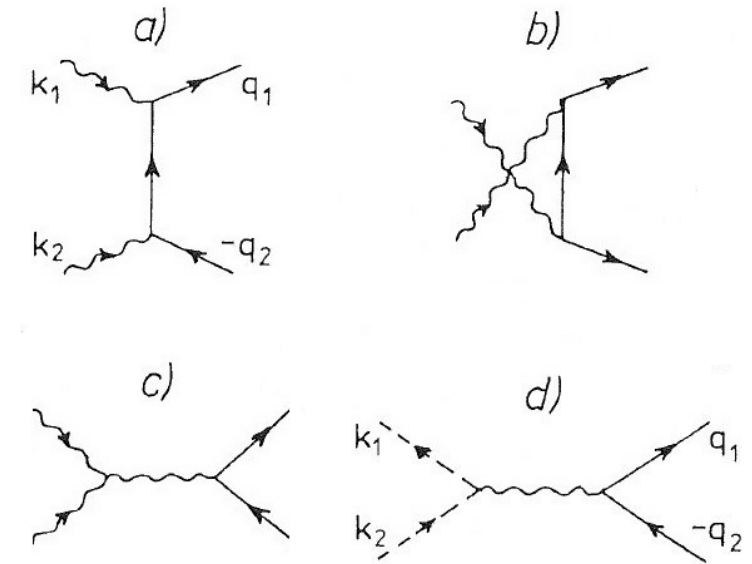

Fig. 5. Lowest order QCD diagrams for $s \bar{s}$ production: a,b,c) $g g \rightarrow s \bar{s}$, and d) $q \bar{q} \rightarrow s \bar{s}$.

which leads for $\mu / 3=\mu_{q}<\pi T$ to the following expressions for the entropy per baryon (including the gluonic entropy second $T^{3}$ term in eq. (3.14)):

$$
\frac{\mathcal{S}}{\nu} \approx \frac{37}{15} \pi^{2} \frac{T}{\mu_{q}} \stackrel{T \sim \mu_{q}}{\longleftrightarrow} 25 !
$$

As this simple estimate shows, plasma events are extremely entropy-rich, i.e., they contain very high particle multiplicity. In order to estimate the particle multiplicity, one may simply divide the total entropy created in the collision by the entropy per particle for massless black body radiation, which is $S / n=4$. This suggests that, at $T \sim \mu_{q}$, there are roughly six pions per baryon.

\section{Strange quarks in plasma}

In lowest order in perturbative QCD, $s \bar{s}$ quark pairs can be created by gluon fusion processes, fig. 5a,b,c; and by annihilation of light quark-antiquark pairs, see fig. $5 \mathrm{~d}$. The averaged total cross-sections for these processes were calculated by Brian Combridge [32].

Given the averaged cross-sections, it is easy to calculate the rate of events per unit time, summed over all final and averaged over initial states

$$
\begin{aligned}
\frac{\mathrm{d} N}{\mathrm{~d} t}= & \int \mathrm{d}^{3} x \sum_{i} \int \frac{\mathrm{d}^{3} k_{1} \mathrm{~d}^{3} k_{2}}{(2 \pi)^{3}\left|k_{1}\right|(2 \pi)^{3}\left|k_{2}\right|} \rho_{i, 1}\left(k_{1}, x\right) \rho_{i, 2}\left(k_{2}, x\right) \\
& \times \int_{4 M^{2}}^{\infty} \mathrm{d} s \delta\left(s-\left(k_{1}+k_{2}\right)^{2}\right) k_{1}^{\mu} k_{2 \mu} \bar{\sigma}(s) .
\end{aligned}
$$

The factor $k_{1} \cdot k_{2} /\left|k_{1}\right|\left|k_{2}\right|$ is the relative velocity for massless gluons or light quarks, and we have introduced a dummy integration over $s$ in order to facilitate the calculations. The phase space densities $\rho_{i}(k, x)$ can be approximated by assuming the $x$-independence of temperature $T(x)$ and the chemical potential $\mu(x)$, in the so-called local statistical equilibrium. Since $\rho$ then only depends on the absolute value of $\boldsymbol{k}$ in the rest frame of the equilibrated plasma, we can easily carry out the relevant integrals and obtain for the dominant process of the gluon 
fusion reaction fig. 5a,b,c the invariant rate per unit time and volume [33]:

$\mathcal{A}=\frac{\mathrm{d}^{4} N}{\mathrm{~d}^{3} x \mathrm{~d} t} \approx \mathcal{A}_{g}=\frac{7 \alpha_{s}^{2}}{6 \pi^{2}} M T^{3} \mathrm{e}^{-2 M / T}\left(1+\frac{51}{14} \frac{T}{M}+\ldots\right)$,

where $M$ is the strange quark mass ${ }^{4}$.

The abundance of $s \bar{s}$ pairs cannot grow forever. At some point the $s \bar{s}$ annihilation reaction will restrict the strange quark population. It is important to appreciate that the $s \bar{s}$ pair annihilations may not proceed via the two-gluon channel, but instead occasionally through $\gamma \mathrm{G}$ (photon-Gluon) final states [34]. The noteworthy feature of such a reaction is the production of relatively high energy $\gamma$ 's at an energy of about $700-900 \mathrm{MeV}(T=$ $160 \mathrm{MeV}$ ) stimulated by coherent glue emission. These $\gamma$ 's will leave the plasma without further interactions and provide an independent confirmation of the $s$-abundance in the plasma.

The loss term of the strangeness population is proportional to the square of the density $n_{s}$ of strange and antistrange quarks. With $n_{s}(\infty)$ being the saturation density at large times, the following differential equation determines $n_{s}$ as a function of time [13]

$$
\frac{\mathrm{d} n_{s}}{\mathrm{~d} t} \approx A\left\{1-\left[\frac{n_{s}(t)}{n_{s}(\infty)}\right]^{2}\right\} .
$$

Thus we find

$$
n_{s}(t)=n_{s}(\infty) \frac{\tanh (t / 2 \tau)+\frac{n_{s}(0)}{n_{s}(\infty)}}{1+\frac{n_{s}(0)}{n_{s}(\infty)} \tanh (t / 2 \tau)}, \quad \tau=\frac{n_{s}(\infty)}{2 \mathcal{A}}
$$

where

$$
\tau=\frac{n_{s}(\infty)}{2 \mathcal{A}}
$$

The relaxation time $\tau$ of the strange quark density in eq. (4.5) is obtained using the saturated phase space in eq. (4.5). We have [33]

$$
\tau \approx \tau_{g}=\left(\frac{\pi}{2}\right)^{1 / 2} \frac{9 M^{1 / 2}}{7 \alpha_{s}^{2}} T^{-3 / 2} \mathrm{e}^{M / T}\left(1+\frac{99}{56} \frac{T}{M}+\ldots\right)^{-1} .
$$

For $\alpha_{s} \sim 0.6$ and $M \sim T$, we find from eq. (4.6) that $\tau \sim 4 \times 10^{-23}$ s. $\tau$ falls off rapidly with increasing temperature. Figure 6 shows the approach of $n_{s}(t)$, normalized with baryon density, to the fully saturated phase space as a function of time. For $M \lesssim T=160 \mathrm{MeV}$, the saturation requires $4 \times 10^{-23} \mathrm{~s}$, while for $T=200 \mathrm{MeV}$, we need $2 \times 10^{-23} \mathrm{~s}$, corresponding to the anticipated lifetime of the plasma. But it is important to observe that, even at $T=120 \mathrm{MeV}$, the phase space is half-saturated in

\footnotetext{
${ }^{4}$ In eq. (4.2) a factor 2 was included to reduce the invariant rate $\mathcal{A}$, see Erratum: "Strangeness Production in the QuarkGluon Plasma" Johann Rafelski and Berndt Müller, Phys. Rev. Lett. 56, 2334 (1986). This factor did not carry through to any of the following results. However, additional definition factors "2" show up below in eqs. (4.4), (4.5).
}

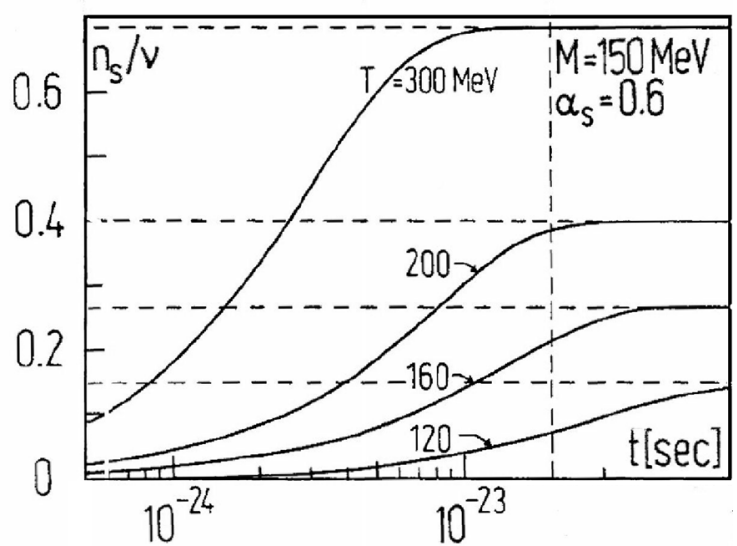

Fig. 6. Time evolution of the strange quark to baryon number abundance in the plasma for various temperatures $T \sim \mu_{q}=$ $\mu / 3 . M=150 \mathrm{MeV}, \alpha_{s}=0.6$.

$2 \times 10^{-23} \mathrm{~s}$, a point to which we will return below. Another remarkable fact is the high abundance of strangeness relative to baryon number seen in fig. 6 - here, baryon number was computed assuming $T \sim \mu_{q}=\mu / 3$ (see eq. (3.15)). These two facts, namely:

1) high relative strangeness abundance in plasma,

2) practical saturation of available phase space,

have led me to suggest the observation of strangeness as a possible signal of quark-gluon plasma [35].

There are two elements in point 1) above: firstly, strangeness in the quark-gluon phase is practically as abundant as the anti-light quarks $\bar{u}=\bar{d}=\bar{q}$, since both phase spaces have similar suppression factors: for $\bar{u}, \bar{d}$ it is the baryon chemical potential, for $s, \bar{s}$ the mass $\left(M \approx \mu_{q}\right)$

$$
\begin{aligned}
\frac{s}{V} & =\frac{\bar{s}}{V}=6 \int \frac{\mathrm{d}^{3} p}{(2 \pi)^{3}} \frac{1}{\mathrm{e}^{\sqrt{p^{2}+M^{2}} / T}+1}, \\
\frac{\bar{q}}{V} & =6 \int \frac{\mathrm{d}^{3} p}{(2 \pi)^{3}} \frac{1}{\mathrm{e}^{|p| / T+\mu_{q} / T}+1} .
\end{aligned}
$$

Note that the chemical potential of quarks suppresses the $\bar{q}$ density. This phenomenon reflects on the chemical equilibrium between $q \bar{q}$ and the presence of a light quark density associated with the net baryon number. Secondly, strangeness in the plasma phase is more abundant than in the hadronic gas phase (even if the latter phase space is saturated) when compared at the same temperature and baryon chemical potential in the phase transition region. The rationale for the comparison at fixed thermodynamic variables, rather than at fixed values of microcanonical variables such as energy density and baryon density, is outlined in the next section. I record here only that the abundance of strangeness in the plasma is well above that in the hadronic gas phase space (by factors 1-6) and the two become equal only when the baryon chemical potential $\mu$ is so large that abundant production of hyperons becomes possible. This requires a hadronic phase at an energy density of $5-10 \mathrm{GeV} / \mathrm{fm}^{3}$. 


\section{How to discover the quark-gluon plasma}

Here only the role of the strange particles in the anticipated discovery will be discussed. My intention is to show that, under different possible transition scenarios, characteristic anomalous strange particle patterns emerge. Examples presented are intended to provide some guidance to future experiments and are not presented here in order to imply any particular preference for a reaction channel. I begin with a discussion of the observable quantities.

The temperature and chemical potential associated with the hot and dense phase of nuclear collision can be connected with the observed particle spectra, and, as discussed here, particle abundances. The last grand canonical variable - the volume - can be estimated from particle interferences. Thus, it is possible to use these measured variables, even if their precise values are dependent on a particular interpretational model, to uncover possible rapid changes in a particular observable. In other words, instead of considering a particular particle multiplicity as a function of the collision energy $\sqrt{s}$, I would consider it as a function of, e.g., mean transverse momentum $\left\langle p_{\perp}\right\rangle$, which is a continuous function of the temperature (which is in turn continuous across any phase transition boundary).

To avoid possible misunderstanding of what I want to say, here I consider the (difficult) observation of the width of the $\mathrm{K}^{+}$two-particle correlation function in momentum space as a function of the average $\mathrm{K}^{+}$ transverse momentum obtained at given $\sqrt{s}$. Most of $\mathrm{K}^{+}$ would originate from the plasma region, which, when it is created, is relatively small, leading to a comparatively large width. (Here I have assumed a first order phase transition with substantial increase in volume as matter changes from plasma to gas.) If, however, the plasma state were not formed, $\mathrm{K}^{+}$originating from the entire hot hadronic gas domain would contribute a relatively large volume which would be seen; thus the width of the two-particle correlation function would be small. Thus, a first order phase transition implies a jump in the $\mathrm{K}^{+}$ correlation width as a function of increasing $\left\langle p_{\perp}\right\rangle_{\mathrm{K}^{+}}$, as determined in the same experiment, varying $\sqrt{s}$.

From this example emerges the general strategy of my approach: search for possible discontinuities in observables derived from discontinuous quantities (such as volume, particle abundances, etc.) as a function of quantities measured experimentally and related to thermodynamic variables always continuous at the phase transition: temperature, chemical potentials, and pressure. This strategy, of course, can only be followed if, as stated in the first sentence of this report, approximate local thermodynamic equilibrium is also established.

Strangeness seems to be particularly useful for plasma diagnosis, because its characteristic time for chemical equilibration is of the same order of magnitude as the expected lifetime of the plasma: $\tau \sim 1-3 \times 10^{-23} \mathrm{~s}$. This means that we are dominantly creating strangeness in the zone where the plasma reaches its hottest stage - freezing over the abundance somewhat as the plasma cools down. However, the essential effect is that the strangeness abundance in the plasma is greater, by a factor of about 30, than that expected in the hadronic gas phase at the same values of $\mu, T$. Before carrying this further, let us note that, in order for strangeness to disappear partially during the phase transition, we must have a slow evolution, with time constants of $\sim 10^{-22} \mathrm{~s}$. But even so, we would end up with strangeness-saturated phase space in the hadronic gas phase, i.e., roughly ten times more strangeness than otherwise expected. For similar reasons, i.e., in view of the rather long strangeness production time constants in the hadronic gas phase, strangeness abundance survives practically unscathed in this final part of the hadronization as well. Facit:

if a phase transition to the plasma state has occurred, then on return to the hadron phase, there will be most likely significantly more strange particles around than there would be (at this $T$ and $\mu$ ) if the hadron gas phase had never been left.

In my opinion, the simplest observable proportional to the strange particle multiplicity is the rate of $\mathrm{V}$-events from the decay of strange baryons $(e . g ., \Lambda)$ and mesons (e.g., $\mathrm{K}_{s}$ ) into two charged particles. Observations of this rate require a visual detector, e.g., a streamer chamber. To estimate the multiplicity of V-events, I reduce the total strangeness created in the collision by a factor $1 / 3$ to select only neutral hadrons and another factor $1 / 2$ for charged decay channels. We thus have

$$
\left\langle n_{\mathrm{V}}\right\rangle \approx \frac{1}{6} \frac{\langle s\rangle+\langle\bar{s}\rangle}{\langle b\rangle}\langle b\rangle \sim \frac{\langle b\rangle}{15},
$$

where I have taken $\langle s\rangle /\langle b\rangle \sim 0.2$ (see fig. 6). Thus for events with a large baryon number participation, we can expect to have several V's per collision, which is 100-1000 times above current observation for $\mathrm{Ar}-\mathrm{KCl}$ collision at 1.8 GeV/Nuc kinetic energy [36].

Due to the high $\bar{s}$ abundance, we may further expect an enrichment of strange antibaryon abundances [35]. I would like to emphasize here $\bar{s} \bar{s} \bar{q}$ states (anticascades) created by the accidental coagulation of two $\bar{s}$ quarks helped by a gluon $\rightarrow \bar{q}$ reaction. Ultimately, the $\bar{s} \bar{s} \bar{q}$ states become $\bar{s} \bar{q} \bar{q}$, either through an $\bar{s}$ exchange reaction in the gas phase or via a weak interaction much, much later. However, half of the $\bar{s} \bar{q} \bar{q}$ states are then visible as $\bar{\Lambda}$ decays in a visual detector. This anomaly in the apparent $\bar{\Lambda}$ abundance is further enhanced by relating it to the decreased abundance of antiprotons, as described above.

Unexpected behavior of the plasma-gas phase transition can greatly influence the channels in which strangeness is found. For example, in an extremely particle-dense plasma, the produced $s \bar{s}$ pairs may stay near to each other - if a transition occurs without any dilution of the density, then I would expect a large abundance of $\phi(1020) s \bar{s}$ mesons, easily detected through their partial decay mode $(1 / 4 \%)$ to a $\mu^{+} \mu^{-}$pair.

Contrary behavior will be recorded if the plasma is cool at the phase transition, and the transition proceeds slowly - major coagulation of strange quarks can then be expected with the formation of sss and $\bar{s} \bar{s} \bar{s}$ baryons and in general $(s)^{3 n}$ clusters. Carrying this even further, supercooled plasma may become "strange" nuclear (quark) 
matter [37]. Again, visual detectors will be extremely successful here, showing substantial decay cascades of the same heavy fragment.

In closing this discussion, I would like to give warning about the pions. From the equations of state of the plasma, we have deduced in sect. 3 a very high specific entropy per baryon. This entropy can only increase in the phase transition and it leads to very high pion multiplicity in nuclear collisions, probably created through pion radiation from the plasma $[19,20]$ and sequential decays. Hence by relating anything to the pion multiplicity, e.g., considering $\mathrm{K} / \pi$ ratios, we dilute the signal from the plasma. Furthermore, pions are not at all characteristic for the plasma; they are simply indicating high entropy created in the collision. However, we note that the $\mathrm{K} / \pi$ ratio can show substantial deviations from values known in $p p$ collisions - but the interpretations of this phenomenon will be difficult.

It is important to appreciate that the experiments discussed above would certainly be quite complementary to the measurements utilizing electromagnetically interacting probes, e.g., dileptons, direct photons. Strangenessbased measurements have the advantage that they have much higher counting rates than those recording electromagnetic particles.

1983: I would like to thank R. Hagedorn, B. Müller, and P. Koch for fruitful and stimulating discussions, and R. Hagedorn for a thorough criticism of this manuscript. Thie work was in part supported by Deutsche Forschungsgemeinschaft. 2015: Also in part supported by the US Department of Energy, Office of Science, Office of Nuclear Physics under award number DE-FG02-04ER41318.

Open Access This is an open access article distributed under the terms of the Creative Commons Attribution License (http://creativecommons.org/licenses/by/4.0), which permits unrestricted use, distribution, and reproduction in any medium, provided the original work is properly cited.

\section{References}

1. B.A. Freedman, L.C. McLerran, Phys. Rev. D 16, 1169 (1977).

2. S.A. Chin, Phys. Lett. B 78, 552 (1978).

3. P.D. Morley, M.B. Kislinger, Phys. Rep. 51, 63 (1979).

4. J.I. Kapusta, Nucl. Phys. B 148, 461 (1978).

5. O.K. Kalashnikov, V.V. Kilmov, Phys. Lett. B 88, 328 (1979).

6. E.V. Shuryak, Phys. Lett. B 81, 65 (1979).

7. E.V. Shuryak, Phys. Rep. 61, 71 (1980).

8. J. Rafelski, R. Hagedorn, From hadron gas to quark matter II, in Thermodynamics of Quarks and Hadrons, edited by H. Satz (North Holland, Amsterdam, 1981).

9. J. Rafelski, M. Danos, Perspectives in high energy nuclear collisions, NBS-IR-83-2725; US Department of Commerce, National Technical Information Service (NTIS) Accession Number PB83-223982 (1983).

10. H. Satz, Phys. Rep. 88, 321 (1982).

11. R. Hagedorn, Suppl. Nuovo Cimento 3, 147 (1964).

12. R. Hagedorn, Nuovo Cimento 6, 311 (1968).
13. R. Hagedorn, How to Deal with Relativistic Heavy Ion Collisions, invited lecture at Quark Matter 1: Workshop on Future Relativistic Heavy Ion Experiments at the Gesellschaft für Schwerionenforschung (GSI), Darmstadt, Germany, 7-10 October 1980; circulated in the GSI816, Orange Report, pp. 282-324, edited by R. Bock, R. Stock, reprinted in Chapter 26 of Melting Hadrons, Boiling Quarks: From Hagedorn temperature to ultra-relativistic heavy-ion collisions at CERN; with a tribute to Rolf Hagedorn, edited by J. Rafelski (Springer, Heidelberg, 2015).

14. R. Hagedorn, J. Rafelski, Phys. Lett. B 97, 136 (1980) see also [8].

15. R. Hagedorn, On a Possible Phase Transition Between Hadron Matter and Quark-Gluon Matter (CERN preprint TH 3392 (1982)), Chapter 24 in L. Rafelski (Editor) loc. cit. (see ref. [13]).

16. R. Hagedorn, Z. Phys. C 17, 265 (1983).

17. R. Hagedorn, I. Montvay, J. Rafelski, Hadronic Matter at Extreme Energy Density, edited by N. Cabibbo (Plenum Press, New York, 1980).

18. J. Rafelski, R. Hagedorn, Thermodynamics of hot nuclear matter in the statistical bootstrap model - 1978, Chapter 23 in J. Rafelski (Editor) loc. cit. (see ref. [13]).

19. J. Rafelski, M. Danos, Pion radiation by hot quark-gluon plasma, CERN preprint TH 3607 (1983) in Proceedings of Sixth High Energy Heavy Ion Study held 28 June - 1 July 1983 at LBNL, CONF-830675, Report LBL-16281 and UC34C (Berkeley, CA, 1983) pp. 515-518.

20. M. Danos, J. Rafelski, Phys. Rev. D 27, 671 (1983).

21. P. Koch, J. Rafelski, W. Greiner, Phys. Lett. B 123, 151 (1983).

22. P. Koch, Diploma thesis, Universität Frankfurt (1983) (published as [21]).

23. A.Z. Mekjian, Nucl. Phys. B 384, 492 (1982).

24. See, e.g., S. Gasiorowicz, J.L. Rosner, Am. J. Phys. 49, 954 (1981).

25. P. Langacker, H. Pagels, Phys. Rev. D 19, 2070 (1979) and references therein.

26. S. Narison, N. Paver, E. de Rafael, D. Treleani, Nucl. Phys. B 212, 365 (1983).

27. A. Chodos, R.L. Jaffe, K. Johnson, C.B. Thorn, V.F. Weisskopf, Phys. Rev. D 9, 3471 (1974).

28. K. Johnson, Acta Phys. Polon. B 6, 865 (1975).

29. T. De Grand, R.L. Jaffe, K. Johnson, J. Kiskis, Phys. Rev. D 12, 2060 (1975).

30. H.Th. Elze, W. Greiner, J. Rafelski, J. Phys. G 6, L149 (1980).

31. J. Rafelski, H.Th. Elze, R. Hagedorn, Hot hadronic and quark matter in $\bar{p}$ annihilation on nuclei, CERN preprint TH 2912 (1980), in Proceedings of Fifth European Symposium on Nucleon-Antinucleon Interactions, Bressanone, 1980 (CLEUP, Padua, 1980) pp. 357-382.

32. B.L. Combridge, Nucl. Phys. B 151, 429 (1979).

33. J. Rafelski, B. Müller, Phys. Rev. Lett. 48, 1066 (1982).

34. G. Staadt, Diploma thesis, Universität Frankfurt (1983) (later published in: Phys. Rev. D 33, 66 (1986)).

35. J. Rafelski, Eur. Phys. J. A 51, 115 (2015) Originally printed in GSI81-6 Orange Report, pp. 282-324, Extreme States of Nuclear Matter - 1980, edited by R. Bock, R. Stock.

36. J.W. Harris, A. Sandoval, R. Stock et al., Phys. Rev. Lett. 47, 229 (1981).

37. S.A. Chin, A.K. Kerman, Phys. Rev. Lett. 43, 1292 (1979). 